\title{
THE CAUCHY PROBLEM FOR STRICTLY HYPERBOLIC OPERATORS WITH NON-ABSOLUTELY CONTINUOUS COEFFICIENTS
}

\author{
By \\ Massimo CiCognANI
}

\section{Introduction}

Let us consider the Cauchy problem

$$
\begin{gathered}
P u(t, x)=0 \quad \text { in }[0, T] \times \boldsymbol{R}^{n} \\
u(0, x)=u_{0}(x), \quad \partial_{t} u(0, x)=u_{1}(x) \quad \text { in } \boldsymbol{R}^{n}
\end{gathered}
$$

for a strictly hyperbolic operator

$$
P=\partial_{t}^{2}-\sum_{j, k=1}^{n} a_{j, k}(t, x) \partial_{x_{j}} \partial_{x_{k}}+\sum_{j=1}^{n} b_{j}(t, x) \partial_{x_{j}}+b_{n+1}(t, x)
$$

with $\left(a_{j, k}\right)$ a real symmetric matrix, $b_{j} \in C\left([0, T] ; \mathscr{B}\left(\boldsymbol{R}^{n}\right)\right), \mathscr{B}\left(\boldsymbol{R}^{n}\right)$ the space of all $C^{\infty}$ functions which are bounded together with all their derivatives in $\boldsymbol{R}^{n}$.

It is well known that if $\partial_{t} a_{j, k} \in L^{1}\left([0, T] ; \mathscr{B}\left(\boldsymbol{R}^{n}\right)\right)$ then problem (1) is well posed in Sobolev spaces: for every $u_{0} \in H^{s}\left(\boldsymbol{R}^{n}\right), u_{1} \in H^{s-1}\left(\boldsymbol{R}^{n}\right)$ there is a unique solution $u \in C\left([0, T] ; H^{s}\left(\boldsymbol{R}^{n}\right)\right) \cap C^{1}\left([0, T] ; H^{s-1}\left(\boldsymbol{R}^{n}\right)\right)$ which satisfies

$$
\|u(t)\|_{s}+\left\|\partial_{t} u(t)\right\|_{s-1} \leq C\left(\left\|u_{0}\right\|_{s}+\left\|u_{1}\right\|_{s-1}\right), \quad 0 \leq t \leq T .
$$

By the finite speed of propagation one obtains the well posedness in $C^{\infty}$.

Our aim is to consider non-absolutely continuous coefficients assuming $a_{j, k} \in$ $\left.\left.C^{1}(] 0, T\right] ; \mathscr{B}\left(\boldsymbol{R}^{n}\right)\right)$ and

$$
\left|\partial_{t} a_{j, k}(t, x)\right| \leq C t^{-q}, \quad q \geq 1, t>0, x \in \boldsymbol{R}^{n}
$$

as it is done by Colombini, Del Santo and Kinoshita in [3] for coefficients of $P$ depending only on the time variable $t$. Here we treat the general case and, beside (4), we permit: 


$$
\left|\partial_{x}^{\beta} a_{j, k}(t, x)\right| \leq C_{\beta} t^{-p}, \quad p \in\left[0,1\left[,|\beta|>0, t>0, x \in \boldsymbol{R}^{n} .\right.\right.
$$

For $q=1$ in (4) and any $p \in[0,1[$ in (5), we prove the inequality

(6) $\|u(t)\|_{s-h}+\left\|\partial_{t} u(t)\right\|_{s-1-h} \leq C\left(\|u(0)\|_{s}+\left\|\partial_{t} u(0)\right\|_{s-1}\right), \quad C, h>0,0 \leq t \leq T$

for every $u \in C\left([0, T] ; H^{s+1}\left(R^{n}\right)\right) \cap C^{1}\left([0, T] ; H^{s}\left(R^{n}\right)\right)$ such that $P u=0$. In particular, we obtain the well posedness in $C^{\infty}$ of the Cauchy problem (1) with a loss of $h$ derivatives.

In the case $q>1$ in (4), we assume boundness and Gevrey regularity $\gamma^{(s)}$ for the coefficients, that is we take $p=0$ and $C_{\beta}=C A^{|\beta|}(\beta !)^{s}$ in (5). Then we prove the well posedness of problem (1) in $\gamma^{(s)}$ for $1<s<q /(q-1)$.

We refer to [3] for counter examples that show the sharpness of these results; in particular $C^{\infty}$ well posedness does not hold for $q>1$.

In (4) and (5) one can subsitute $t^{-q}$ and $t^{-p}$ with $\left|T_{0}-t\right|^{-q}$ and $\left|T_{0}-t\right|^{-p}$, respectively, $T_{0} \in[0, T], t \neq T_{0}$. So inequality (6) can be applied also to the study of the blowup rate in some nonlinear equations. Consider, for istance, a smooth solution $u$ for $t<T$ of

$$
\partial_{t}^{2} u-\alpha\left(\int_{0}^{t} \partial_{x} u(s, x) d s\right) \partial_{x}^{2} u=0, \quad \alpha(y) \geq \alpha_{0}>0
$$

such that

$$
\left|\partial_{x}^{\beta} u(t, x)\right| \leq C_{\beta}(T-t)^{-1}, \quad t<T .
$$

If $\alpha^{\prime}$ is bounded and $\left|\alpha^{(k)}(y)\right| \leq A_{k} e^{\mu|y|}, \quad \mu<1 / C_{1}, \quad k \geq 2$, then $a(t, x):=$ $\alpha\left(\int_{0}^{t} \partial_{x} u(s, x) d s\right)$ satisfies (4) with $q=1$ and (5) with $\left.p \in\right] \mu C_{1}, 1\left[,(T-t)^{-1}\right.$ and $(T-t)^{-p}$ in place of $t^{-1}$ and $t^{-p}$ respectively. So inequality (6) implies $u \in C^{\infty}$ also for $t=T$. This means that $(T-t)^{-1}$ is not a sufficient breakdown rate of the derivatives $\partial_{x}^{\beta} u$ to have blowup of $u$ at $t=T$, cf. [1].

\section{Main Results}

Let

$$
P=\partial_{t}^{2}-\sum_{j, k=1}^{n} a_{j, k}(t, x) \partial_{x_{j}} \partial_{x_{k}}+\sum_{j=1}^{n} b_{j}(t, x) \partial_{x_{j}}+b_{n+1}(t, x)
$$

be a linear differential operator in $[0, T] \times \boldsymbol{R}^{n}$, with $\left(a_{j, k}\right)$ a symmetric matrix of real valued functions, $\left.\left.a_{j, k} \in C^{1}(] 0, T\right] ; C^{\infty}\left(\boldsymbol{R}^{n}\right)\right), b_{j} \in C\left([0, T] ; C^{\infty}\left(\boldsymbol{R}^{n}\right)\right)$. We consider the Cauchy problem for the equation 


$$
P u(t, x)=0 \quad \text { in }[0, T] \times R^{n}
$$

with initial data at $t=0$

$$
u(0, x)=u_{0}(x), \quad \partial_{t} u(0, x)=u_{1}(x) \quad \text { in } \boldsymbol{R}^{n}
$$

under the hypothesis of strict hyperbolicity

$$
a(t, x, \xi):=\sum_{j, k=1}^{n} a_{j, k}(t, x) \xi_{j} \xi_{k} \geq c_{0}|\xi|^{2}, \quad c_{0}>0
$$

and we deal with its well posedness according to the behaviour of $\partial_{t} a$ as $t \rightarrow 0$. Our first result is the following:

THEOREM 1. Assume that there exist $p, r \in\left[0,1\left[\right.\right.$ and positive constants $C_{\beta}$ such that

$$
\left|\partial_{x}^{\beta} a_{j, k}(t, x)\right| \leq C_{\beta} t^{-p}, \quad|\beta|>0 ; \quad\left|\partial_{x}^{\beta} \partial_{t} a_{j, k}(t, x)\right| \leq C_{\beta} t^{-1-r|\beta|}, \quad|\beta| \geq 0 .
$$

Then, for every $u_{0}, u_{1} \in C^{\infty}\left(\boldsymbol{R}^{n}\right)$ the Cauchy problem (1.1), (1.2) has a unique solution $u \in C^{1}\left([0, T] ; C^{\infty}\left(\boldsymbol{R}^{n}\right)\right)$.

REMARK. A consequence of (1.4) is the finite speed of propagation. So it is not restrective to consider $u_{0}, u_{1} \in C_{0}^{\infty}\left(\boldsymbol{R}^{n}\right)$ and to assume

$$
\left|\partial_{x}^{\beta} b_{j}(t, x)\right| \leq C_{\beta}, \quad(t, x) \in[0, T] \times \boldsymbol{R}^{n} .
$$

In Section 2 we shall prove an estimate in Sobolev spaces that implies Theorem 1:

THEOREM 2. Under the hypotheses of Theorem 1 there are positive constants $C, h$ such that for every $u \in C\left([0, T] ; H^{s+1}\left(\boldsymbol{R}^{n}\right)\right) \cap C^{1}\left([0, T] ; H^{s}\left(\boldsymbol{R}^{n}\right)\right)$ which satisfies $P u=0$ we have

$$
\|u(t)\|_{s-h}+\left\|\partial_{t} u(t)\right\|_{s-1-h} \leq C\left(\|u(0)\|_{s}+\left\|\partial_{t} u(0)\right\|_{s-1}\right), \quad 0 \leq t \leq T .
$$

When $t \partial_{t} a\left(t, x, \xi|\xi|^{-2}\right)$ is not bounded, problem (1.1), (1.2) may not be well posed in $C^{\infty}$.

For $s>1, A>0$, we denote by $\gamma_{A}^{(s)}=\gamma_{A}^{(s)}\left(\boldsymbol{R}^{n}\right)$ the space of all functions $f$ satisfying

$$
\|f\|_{s, A}:=\sup _{\beta \in Z_{+}^{n}, x \in R^{n}} A^{-|\beta|}(\beta !)^{-s}\left|\partial_{x}^{\beta} f(x)\right|<\infty
$$

so $\gamma^{(s)}:=\bigcup_{A>0} \gamma_{A}^{(s)}$ is a Gevrey space. 
THEOREM 3. Assume $a_{j, k}, b_{j} \in C\left([0, T] ; \gamma_{A}^{(s)}\left(\boldsymbol{R}^{n}\right)\right)$ and

$$
\left.\left.\left|\partial_{x}^{\beta} \partial_{t} a_{j, k}(t, x)\right| \leq C t^{-q} A^{|\beta|}(\beta !)^{s}, \quad(t, x) \in\right] 0, T\right] \times \boldsymbol{R}^{n}, \quad q>1, s<q /(q-1) .
$$

Then there exists $A_{0}>A$ such that for every $u_{0}, u_{1} \in \gamma_{A}^{(s)}\left(\boldsymbol{R}^{n}\right)$ the Cauchy problem (1.1), (1.2) has a unique solution $u \in C^{1}\left([0, T] ; \gamma_{A_{0}}^{(s)}\left(\boldsymbol{R}^{n}\right)\right)$.

As Theorem 1, we shall obtain Theorem 3 from an a priori estimate; so we introduce Gevrey-Sobolev spaces adapted to our problem. We fix $\delta \in] 0,1[$ such that $1 / s=(q-1+\delta) / q$ then for $k>0, t \in[0, T], \mu \in \boldsymbol{R}$ we denote by $H^{k, t, \mu}\left(\boldsymbol{R}^{n}\right)$ the space of all functions $f$ such that:

$$
\|f\|_{k, t, \mu}:=\left\|\exp \left(\frac{k}{\delta}\left(T^{\delta}-t^{\delta}\right)\left\langle D_{x}\right\rangle^{1 / s}\right) f\right\|_{\mu}<\infty,
$$

$\|g\|_{\mu}$ the norm of $g$ in the usual Sobolev space $H^{\mu}\left(\boldsymbol{R}^{n}\right)$.

From Paley-Wiener theorem it follows that

$$
\|f\|_{k, t, \mu} \leq C\|f\|_{s, A}, \quad f \in \gamma_{A}^{(s)}\left(\boldsymbol{R}^{n}\right) \cap C_{0}^{\infty}\left(\boldsymbol{R}^{n}\right), \quad 0 \leq k T^{\delta} / \delta \leq T_{0}
$$

with $T_{0}$ and $C$ positive constants depending on $A$. Conversely, for every $T_{1}<T$ and $k>0$ there is $A_{1}>0$ such that

$$
H^{k, t, \mu}\left(\boldsymbol{R}^{n}\right) \subset \gamma_{A}^{(s)}\left(\boldsymbol{R}^{n}\right), \quad t \in\left[0, T_{1}\right], A>A_{1}, \mu>n / 2 .
$$

For functions $u(t, x)$ we define the space

$$
\begin{aligned}
C_{T}^{j}\left(H^{k, t, \mu}\right):= & \left\{u ; t \rightarrow \exp \left(\frac{k}{\delta}\left(T^{\delta}-t^{\delta}\right)\left\langle D_{x}\right\rangle^{1 / s}\right) \partial_{t}^{h} u(t, \cdot)\right. \text { is continuous from } \\
& {\left.[0, T] \text { to } H^{\mu-h}\left(\boldsymbol{R}^{n}\right), h=0, \ldots, j\right\} . }
\end{aligned}
$$

THEOREM 4. Under the hypotheses of Theorem 3 there are positive constants $k_{0}, T_{0}, C$ such that for every $u \in C_{T}^{1}\left(H^{k, t, \mu+1}\right), k T^{\delta} / \delta \leq T_{0}, k \geq k_{0}$, which satisfies $P u=0$ we have

$$
\|u(t)\|_{k, t, \mu}+\left\|\partial_{t} u(t)\right\|_{k, t, \mu-1} \leq C\left(\|u(0)\|_{k, 0, \mu}+\left\|\partial_{t} u(0)\right\|_{k, 0, \mu-1}\right), \quad 0 \leq t \leq T
$$

We shall prove Theorem 4 in Section 3. From estimate (1.8) we can solve problem (1.1), (1.2) in $\left[0, T_{1}\right], T_{1}=\left(\delta T_{0} / k_{0}\right)^{1 / \delta}$. This is sufficient to prove Theorem 3 since we have $a_{j, k} \in C^{1}\left(\left[T_{1}, T\right] ; \gamma_{A}^{(s)}\left(\boldsymbol{R}^{n}\right)\right)$ that ensures $\gamma^{(s)}$ well posedness in $\left[T_{1}, T\right]$. 


\section{2. $C^{\infty}$ Well Posedness}

In this section we prove Theorem 2 which implies Theorem 1.

Writing

$$
\begin{gathered}
P=\partial_{t}^{2}+a\left(t, x, D_{x}\right)+b\left(t, x, D_{x}\right), \quad D_{x}=\frac{1}{i} \partial_{x} \quad(i=\sqrt{-1}) \\
a(t, x, \xi)=\sum_{j, k=1}^{n} a_{j, k}(t, x) \xi_{j} \xi_{k}, \quad b(t, x, \xi)=i \sum_{j=1}^{n} b_{j}(t, x) \xi_{j}+b_{n+1}(t, x),
\end{gathered}
$$

the assumptions on $P$ are the following:

$$
\begin{aligned}
& a(t, x, \xi) \geq c_{0}|\xi|^{2}, \quad c_{0}>0, \\
& \left|\partial_{x}^{\beta} \partial_{\xi}^{\alpha} a(t, x, \xi)\right| \leq C_{\alpha, \beta} t^{-p}\langle\xi\rangle^{2-|\alpha|}, \quad|\alpha| \geq 0,|\beta|>0, \\
& \left|\partial_{x}^{\beta} \partial_{\xi}^{\alpha} \partial_{t} a(t, x, \xi)\right| \leq C_{\alpha, \beta} t^{-1-r|\beta|}\langle\xi\rangle^{2-|\alpha|}, \quad|\alpha| \geq 0,|\beta| \geq 0, \\
& \left|\partial_{x}^{\beta} \partial_{\xi}^{\alpha} b(t, x, \xi)\right| \leq C_{\alpha, \beta}\langle\xi\rangle^{1-|\alpha|}, \quad|\alpha| \geq 0,|\beta| \geq 0,
\end{aligned}
$$

$p, r \in[0,1[,(t, x, \xi) \in] 0, T] \times \boldsymbol{R}^{n} \times \boldsymbol{R}^{n},\langle\xi\rangle=\left(1+|\xi|^{2}\right)^{1 / 2}$.

In particular (2.3) gives also

$$
\left|\partial_{\xi}^{\alpha} a(t, x, \xi)\right| \leq C_{\alpha} \log (1+1 / t)\langle\xi\rangle^{2-|\alpha|}, \quad|\alpha| \geq 0 .
$$

We modify the symbol $a$ for $\langle\xi\rangle \leq 2 / t$ defining

$$
\begin{aligned}
& a_{0}(t, x, \xi)=\varphi(t\langle\xi\rangle)\langle\xi\rangle^{2}+(1-\varphi(t\langle\xi\rangle)) a(t, x, \xi) \\
& \qquad \in C^{\infty}(\boldsymbol{R}), \quad 0 \leq \varphi \leq 1, \quad \varphi=1 \text { in }[0,1], \quad \varphi=0 \text { in }[2,+\infty[.
\end{aligned}
$$

Then $\lambda(t)=\sqrt{a_{0}(t)}, 0 \leq t \leq T$, is a family of symbols of pseudodifferential operators in $\boldsymbol{R}^{n}$ which satisfies

$$
\begin{gathered}
\lambda(t, x, \xi) \geq c\langle\xi\rangle, \quad c>0 \\
\left|\partial_{x}^{\beta} \partial_{\xi}^{\alpha} \lambda(t, x, \xi)\right| \leq C_{\alpha, \beta}\langle\xi\rangle^{1-|\alpha|}\left[1+H(t\langle\xi\rangle) t^{-p|\beta|}(\log (1+1 / t))^{1+|\alpha|}\right], \\
H(y)=0 \text { for } y<1, \quad H(y)=1 \text { for } y \geq 1 .
\end{gathered}
$$

In particular, if we denote as usual by $S_{\rho, \delta}^{m}$ the class of all symbols $q(x, \xi)$ such that $\left|\partial_{x}^{\beta} \partial_{\xi}^{\alpha} q(x, \xi)\right| \leq C_{\alpha, \beta}\langle\xi\rangle^{m-\rho|\alpha|+\delta|\beta|}, 0 \leq \delta<\rho \leq 1$, we have that $\{\lambda(t)$; $0 \leq t \leq T\}$ is bounded in $S_{\rho, p}^{1+\varepsilon}$ and $\left\{\lambda^{-1}(t) ; 0 \leq t \leq T\right\}$ is bounded in $S_{\rho, p}^{-1}$ for every $\varepsilon>0$ and every $\rho \in] p, 1[$.

Another consequence is that the symbol $r(t, x, \xi)$ of the operator $a_{0}-\lambda^{2}$ verifies $t^{1-\varepsilon} r \in C\left([0, T] ; S_{1, p}^{1}\right)$ for every $\left.\varepsilon \in\right] 0,1-p[$. 
From (2.2), (2.3) and (2.5) we get:

$$
\begin{aligned}
& \left|\partial_{x}^{\beta} \partial_{\xi}^{\alpha} \partial_{t} \lambda(t, x, \xi)\right| \leq C_{\alpha, \beta}\langle\xi\rangle^{1-|\alpha|} H(t\langle\xi\rangle) t^{-1-\delta|\beta|}(\log (1+1 / t))^{1+|\alpha|} \\
& \delta=\max \{p, r\}
\end{aligned}
$$

which implies the boundness in $S_{1, \delta}^{1+\varepsilon^{\prime}}$ of $\left\{t^{1-\varepsilon} \partial_{t} \lambda(t) ; 0 \leq t \leq T\right\}$ for any given $\varepsilon \in] 0,1\left[, \varepsilon^{\prime} \in\right] \varepsilon, 1\left[\right.$, by using $t^{-\varepsilon}(\log (1+1 / t))^{1+|\alpha|} \leq C_{\alpha} t^{-\varepsilon^{\prime}} \leq C_{\alpha}\langle\xi\rangle^{\varepsilon^{\prime}}$ on the support of $H(t\langle\xi\rangle)$.

Now we factorize the principal part of the operator $P=\partial_{t}^{2}+a+b$ :

$$
\begin{aligned}
& P=\left(\partial_{t}-i \lambda\right)\left(\partial_{t}+i \lambda\right)+a-a_{0}+a_{1}, \\
& a_{1}=-i\left[\partial_{t}, \lambda\right]+a_{0}-\lambda^{2}+b .
\end{aligned}
$$

Obviously $t^{p+m}\left(a(t)-a_{0}(t)\right), 0 \leq t \leq T$, is a bounded and continuous family in $S_{1,0}^{2-m}$ for any $m \geq 0$ while $t^{1-\varepsilon} a_{1}(t), 0 \leq t \leq T$ is bounded and continuous in $S_{1, \delta}^{1+\varepsilon^{\prime}}$ for every $\left.\varepsilon \in\right] 0,1-\delta\left[, \varepsilon^{\prime} \in\right] \varepsilon, 1\left[\right.$. Hereafter we fix $0<\varepsilon<\varepsilon^{\prime}<1-\delta$.

We have not $a-a_{0}+a_{1} \in L^{1}\left([0, T] ; S_{\rho, \delta}^{1}\right)$ that by Gronwall's method would give the classical energy inequality

$$
\|u(t)\|_{s}+\left\|\partial_{t} u(t)\right\|_{s-1} \leq C\left(\|u(0)\|_{s}+\left\|\partial_{t} u(0)\right\|_{s-1}\right), \quad C>0,0 \leq t \leq T
$$

for every $u \in C\left([0, T] ; H^{s+1}\left(\boldsymbol{R}^{n}\right)\right) \cap C^{1}\left([0, T] ; H^{s}\left(\boldsymbol{R}^{n}\right)\right)$ such that $P u=0$.

Anyway a weaker condition in this direction holds true: $a-a_{0}=$ $\varphi(t\langle\xi\rangle)\left(a-\langle\xi\rangle^{2}\right)$ is bounded by $C\langle\xi\rangle^{2} \log (1+1 / t)$ and vanishes for $t\langle\xi\rangle>2$ so we can find a smooth function $\psi_{0}(t, \xi)$ such that

$$
\left|a-a_{0}\right|\langle\xi\rangle^{-1} \leq \psi_{0}, \quad(\log (1+1 / t))^{-1} \psi_{0} \in C\left([0, T] ; S_{1,0}^{1}\right)
$$

and

$$
\int_{0}^{T}\left|\partial_{\xi}^{\alpha} \psi_{0}(t, \xi)\right| d t \leq C_{\alpha}\langle\xi\rangle^{1-|\alpha|} \int_{0}^{2 /\langle\xi\rangle} \log (1+1 / t) d t \leq h_{\alpha}\langle\xi\rangle^{-|\alpha|} \log (1+\langle\xi\rangle)
$$

Concerning $a_{1}$ we have that

$$
\partial_{t} \lambda=(2 \lambda)^{-1}\left[\langle\xi\rangle \varphi^{\prime}(t\langle\xi\rangle)\left(\langle\xi\rangle^{2}-a\right)+(1-\varphi(t\langle\xi\rangle)) \partial_{t} a\right]
$$

is bounded by $C\langle\xi\rangle^{2} \log (1+1 / t)$ for $t\langle\xi\rangle \leq 2$ and by $t^{-1}\langle\xi\rangle$ for $t\langle\xi\rangle>2$ while the symbol of $a_{0}-\lambda^{2}+b$ is bounded by $C t^{-1+\varepsilon}\langle\xi\rangle$. So we can find $\psi_{1}(t, \xi)$ such that

$$
\left|a_{1}\right|\langle\xi\rangle^{-1} \leq \psi_{1}, \quad t^{1-\varepsilon} \psi_{1} \in C\left([0, T] ; S_{1,0}^{\varepsilon^{\prime}}\right)
$$


and

$$
\begin{aligned}
\int_{0}^{T}\left|\partial_{\xi}^{\alpha} \psi_{1}(t, \xi)\right| d t & \leq C_{\alpha}\langle\xi\rangle^{-|\alpha|}\left[1+\langle\xi\rangle \int_{0}^{2 /\langle\xi\rangle} \log (1+1 / t) d t+\int_{2 /\langle\xi\rangle}^{T} \frac{1}{t} d t\right] \\
& \leq h_{\alpha}\langle\xi\rangle^{-|\alpha|} \log (1+\langle\xi\rangle) .
\end{aligned}
$$

Now we use the factorization $(2.9)$ to reduce the equation $P u=0$ to a first order system. For $u \in C\left([0, T] ; H^{s+1}\left(\boldsymbol{R}^{n}\right)\right) \cap C^{1}\left([0, T] ; H^{s}\left(\boldsymbol{R}^{n}\right)\right)$ let us define

$$
U={ }^{t}\left(u_{1}, u_{2}\right), \quad u_{1}=\left(\partial_{t}+i \lambda\right) u, \quad u_{2}=\left\langle D_{x}\right\rangle u-m u_{1},
$$

$m$ the operator with symbol $m(t, x, \xi)=\frac{(1-\varphi(t\langle\xi\rangle / 3))\langle\xi\rangle}{2 i \lambda(t, x, \xi)}$ to have $\langle\xi\rangle=2 i \lambda m$ for $t\langle\xi\rangle>6$ and $(\operatorname{supp} m) \cap\left(\operatorname{supp} a-a_{0}\right)=\varnothing$.

Then it is easy to see that the equation $P u=0$ is equivalent to a first order $2 \times 2$ system $L U=0$,

$$
L=\partial_{t}+K\left(t, x, D_{x}\right), \quad K=D+A, \quad A=A_{0}+A_{1},
$$

where

$$
\begin{aligned}
& D=\left(\begin{array}{cc}
-i \lambda & 0 \\
0 & i \lambda
\end{array}\right), \quad t^{p} A_{0} \in C\left([0, T] ; S_{1,0}^{1}\right) \\
& A_{0}(t, x, \xi)=0 \text { for } t\langle\xi\rangle>6, . \quad t^{1-\varepsilon} A_{1} \in C\left([0, T] ; S_{1, \delta}^{\varepsilon^{\prime}}\right)
\end{aligned}
$$

and there are two positive functions $\psi_{0}(t, \xi), \psi_{1}(t, \xi)$ such that:

$$
\begin{aligned}
& \left|A_{0}\right| \leq \psi_{0}, \quad(\log (1+1 / t))^{-1} \psi_{0} \in C\left([0, T] ; S_{1,0}^{1}\right), \\
& \left|A_{1}\right| \leq \psi_{1}, \quad t^{1-\varepsilon} \psi_{1} \in C\left([0, T] ; S_{1,0}^{\varepsilon^{\prime}}\right), \\
& \int_{0}^{T}\left|\partial_{\xi}^{\alpha} \psi(t, \xi)\right| d t \leq h_{\alpha}\langle\xi\rangle^{-|\alpha|} \log (1+\langle\xi\rangle), \quad \psi=\psi_{0}+\psi_{1} .
\end{aligned}
$$

Since it is

$$
C^{-1}\left(\|u(t)\|_{s+1}+\left\|\partial_{t} u(t)\right\|_{s-\varepsilon}\right) \leq\|U(t)\|_{s} \leq C\left(\|u(t)\|_{s+1+\varepsilon}+\left\|\partial_{t} u(t)\right\|_{s}\right), \quad 0 \leq t \leq T,
$$

we prove Theorem 2 by the following result:

THEOREM 2.1. There are positive constants $C, h$ such that for every $U \in$ $C\left([0, T] ; H^{s+1}\left(\boldsymbol{R}^{n}\right)\right) \cap C^{1}\left([0, T] ; H^{s}\left(\boldsymbol{R}^{n}\right)\right)$ which satisfies $L U=0$ we have

$$
\|U(t)\|_{s-h} \leq C\|U(0)\|_{s}, \quad 0 \leq t \leq T .
$$


Proof. It is sufficient to prove (2.13) for $s=0$ since $\left\langle D_{x}\right\rangle^{s} L\left\langle D_{x}\right\rangle^{-s}$ satisfies the same hypotheses as $L$.

We look for lower bounds of the operator $K=D+A$ in (2.10). As it concerns the diagonal part $D$, from (2.7) we have that the symbol $d(t, x, \xi)$ of the operator $D(t)+D^{*}(t)$ satisfies $t^{1-\varepsilon} d \in C\left([0, T] ; S_{1, \delta}^{0}\right)$ so it follows

$$
2 \operatorname{Re}\langle D U(t), U(t)\rangle \geq-C t^{-1+\varepsilon}\langle U(t), U(t)\rangle, \quad C>0
$$

for every $U \in C\left([0, T] ; H^{1}\left(R^{n}\right)\right)$.

Next we make the change of variable

$$
V=w\left(t, D_{x}\right) U, \quad w(t, \xi)=\exp \left(-\int_{0}^{t} \psi(s, \xi) d s\right),
$$

$\psi=\psi_{0}+\psi_{1}$ the function in (2.12). We have

$$
\|U(t)\|_{h_{0}} \leq 2\|V(t)\|_{0}, \quad U(0)=V(0), h_{0}>0,0<t \leq T
$$

and $L U=0$ if and only if $L_{1} V=0$ with

$$
\begin{aligned}
& L_{1}=w L w^{-1}=\partial_{t}+K_{1}\left(t, x, D_{x}\right), \\
& K_{1}=D+(\psi I+A)+R_{1}, \\
& t^{1-\varepsilon}(\log (1+\langle\xi\rangle))^{-1} R_{1} \in C\left([0, T] ; S_{1, \delta}^{0}\right)
\end{aligned}
$$

Now the symbol of $\psi I+A$ satisfies

$$
\begin{array}{lll}
t^{1-\varepsilon}\left(\psi_{0} I+A_{0}\right) \in C\left([0, T] ; S_{1,0}^{1}\right), & \psi_{0} I+\left(A_{0}+A_{0}^{*}\right) / 2 \geq 0 & \text { for large }|\xi|, \\
t^{1-\varepsilon}\left(\psi_{1} I+A_{1}\right) \in C\left([0, T] ; S_{1, \delta}^{\varepsilon^{\prime}}\right), & \psi_{1} I+\left(A_{1}+A_{1}^{*}\right) / 2 \geq 0 & \text { for large }|\xi|, \\
\varepsilon<\varepsilon^{\prime}<1-\delta, \quad \delta=\max \{p, r\}, &
\end{array}
$$

so the sharp Garding inequality gives

$$
2 \operatorname{Re}\langle(\psi I+A) V(t), V(t)\rangle \geq-C t^{-1+\varepsilon}\langle V(t), V(t)\rangle, \quad C>0
$$

for every $V \in C\left([0, T] ; H^{1}\left(R^{n}\right)\right)$.

For the operator $R_{1}$ we have

$$
2 \operatorname{Re}\left\langle R_{1} V(t), V(t)\right\rangle \geq-h_{1} t^{-1+\varepsilon}\left\langle\log \left(1+\left\langle D_{x}\right\rangle\right) V(t), V(t)\right\rangle, \quad h_{1}>0
$$

that leads us to make the further change of variable (cf. [2]):

$$
W=\left(1+\left\langle D_{x}\right\rangle\right)^{-\alpha(t)} V=\left(1+\left\langle D_{x}\right\rangle\right)^{-\alpha(t)} w\left(t, D_{x}\right) U, \quad \alpha(t)=h_{1} t^{\varepsilon} / \varepsilon,
$$

$h_{1}$ the constant in (2.18). It is 


$$
\|U(t)\|_{-h} \leq 2^{\alpha(T)+1}\|W(t)\|_{0}, \quad U(0)=W(0), \quad h=h_{0}+h_{1} T^{\varepsilon} / \varepsilon, \quad 0 \leq t \leq T,
$$

$h_{0}$ the constant in (2.15), and $L U=0$ if and only if $L_{2} W=0$ with

$$
\begin{aligned}
& L_{2}=\left(1+\left\langle D_{x}\right\rangle\right)^{-\alpha(t)} L_{1}\left(1+\left\langle D_{x}\right\rangle\right)^{\alpha(t)}=\partial_{t}+K_{2}\left(t, x, D_{x}\right), \\
& K_{2}=D+(\psi I+A)+\left(h_{1} t^{-1+\varepsilon} \log \left(1+\left\langle D_{x}\right\rangle\right)+R_{1}\right)+R_{2}, \\
& t^{1-\varepsilon} R_{2} \in C\left([0, T] ; S_{1, \delta}^{0}\right) .
\end{aligned}
$$

Now $h_{1} t^{-1+\varepsilon} \log \left(1+\left\langle D_{x}\right\rangle\right)+R_{1}$ is a positive operator by (2.18) while $t^{1-\varepsilon} R_{2}(t)$ is uniformly bounded in $L^{2}\left(\boldsymbol{R}^{n}\right)$ for $0<t \leq T$. From this, (2.14) and (2.17) we get

$$
2 \operatorname{Re}\left\langle K_{2} W(t), W(t)\right\rangle \geq-C t^{-1+\varepsilon}\langle W(t), W(t)\rangle, \quad C>0
$$

for every $W \in C\left([0, T] ; H^{1}\left(\boldsymbol{R}^{n}\right)\right)$, hence

$$
\frac{d}{d t}\|W(t)\|_{0}^{2} \leq C t^{-1+\varepsilon}\|W(t)\|_{0}^{2}
$$

for every $W \in C\left([0, T] ; H^{1}\left(\boldsymbol{R}^{n}\right)\right) \cap C^{1}\left([0, T] ; H^{0}\left(\boldsymbol{R}^{n}\right)\right)$ such that $L_{2} W=0$. This gives

$$
\|W(t)\|_{0}^{2} \leq \exp \left(C t^{\varepsilon} / \varepsilon\right)\|W(0)\|_{0}^{2}
$$

that is (2.13) with $s=0$ by $(2.19)$.

\section{3. $\gamma^{(s)}$ Well Posedness}

In this section we prove Theorem 4 which implies Theorem 3.

We need to introduce a class pseudodifferential operators in Gevrey spaces:

Definition 3.1. For $m \in \boldsymbol{R}, s>1, A>0$ we denote by $\Gamma_{s, A}^{m}$ the space of all symbols $a(x, \xi)$ such that

$$
|a|_{\Gamma_{s, A, l}^{m}}:=\sup _{(x, \xi) \in R^{2 n},|\alpha+\beta| \leq l, \gamma \in Z_{+}^{n}}\left|\partial_{\xi}^{\alpha} \partial_{x}^{\beta+\gamma} a(x, \xi)\right| A^{-|\gamma|}(\gamma !)^{-s}\langle\xi\rangle^{-m+|\alpha|}
$$

is finite for every $l \in Z_{+}$.

Set $a_{\Lambda}\left(x, D_{x}\right)=e^{\Lambda} a\left(x, D_{x}\right) e^{-\Lambda}, \Lambda=k\left\langle D_{x}\right\rangle^{1 / s}$, and denote by

$$
|a|_{S_{l}^{m}}:=\sup _{(x, \xi) \in R^{2 n},|\alpha+\beta| \leq l}\left|\partial_{\xi}^{\alpha} \partial_{x}^{\beta} a(x, \xi)\right|\langle\xi\rangle^{-m+|\alpha|}
$$

the usual norms in $S_{1,0}^{m}$. In [4] Kajitani proved the following result: 
Proposition 3.2. For every $A>0$ there is $T_{0}>0$ such that

$$
|k|<T_{0}, \quad a \in \Gamma_{s, A}^{m} \Rightarrow a_{\Lambda} \in S_{1,0}^{m}, \quad a_{\Lambda}=a+r, \quad r \in S_{1,0}^{m-1+1 / s}
$$

and for every $l \in Z_{+}^{n}$ there are $C_{l}>0$ and $l^{\prime} \in Z_{+}^{n}$ such that

$$
|r|_{S_{l}^{m-1+1 / s}} \leq C_{l}|a|_{\Gamma_{s, A, l^{\prime}}^{m}}
$$

In particular we have that $a\left(x, D_{x}\right), a \in \Gamma_{s, A}^{m}$, is a continuous operator from $H^{k, t, \mu}\left(\boldsymbol{R}^{n}\right)$ to $H^{k, t, \mu-m}\left(\boldsymbol{R}^{n}\right), H^{k, t, \mu}\left(\boldsymbol{R}^{n}\right)=\exp \left(-\frac{k}{\delta}\left(T^{\delta}-t^{\delta}\right)\left\langle D_{x}\right\rangle^{1 / s}\right) H^{\mu}\left(\boldsymbol{R}^{n}\right)$, for $0<k T^{\delta} / \delta \leq T_{0}, 0 \leq t \leq T$.

Now we can begin the proof of Theorem 4. In this section the assumptions on the operator

$$
P=\partial_{t}^{2}+a\left(t, x, D_{x}\right)+b\left(t, x, D_{x}\right)
$$

are the following:

$$
\begin{aligned}
& a(t, x, \xi) \geq c_{0}|\xi|^{2}, \quad c_{0}>0 \\
& a \in C\left([0, T] ; \Gamma_{s, A}^{2}\right) \\
& t^{q} \partial_{t} a, 0<t \leq T, \text { is a continuous and bounded family in } \Gamma_{s, A}^{2} \\
& q>1, s<q /(q-1) \\
& b \in C\left([0, T] ; \Gamma_{s, A}^{1}\right) .
\end{aligned}
$$

Here we define

$$
\begin{aligned}
& a_{0}(t, x, \xi)=\varphi\left(t^{q}\langle\xi\rangle\right)\langle\xi\rangle^{2}+\left(1-\varphi\left(t^{q}\langle\xi\rangle\right)\right) a(t, x, \xi) \\
& \varphi \in C^{\infty}(\boldsymbol{R}), \quad 0 \leq \varphi \leq 1, \quad \varphi=1 \text { in }[0,1], \quad \varphi=0 \text { in }[2,+\infty[
\end{aligned}
$$

and take $\delta>0$ so that $1 / s=(q-1+\delta) / q$ to have

$$
t^{1-\delta}\left(a-a_{0}\right) \in C\left([0, T] ; \Gamma_{s, A}^{1+1 / s}\right)
$$

using $\langle\xi\rangle \leq 2 t^{-q}$ in the support of $\varphi\left(t^{q}\langle\xi\rangle\right)$.

We have also

$$
\lambda=\sqrt{a_{0}} \in C\left([0, T] ; \Gamma_{s, A}^{1}\right), \quad \lambda^{-1} \in C\left([0, T] ; \Gamma_{s, A}^{-1}\right)
$$

and from (3.6) we get

$$
t^{1-\delta} \partial_{t} \lambda \in C\left([0, T] ; \Gamma_{s, A}^{1+1 / s}\right)
$$

by $\langle\xi\rangle \leq 2 t^{-q}$ in $\operatorname{supp} \varphi^{\prime}\left(t^{q}\langle\xi\rangle\right)$ and $t^{-q} \leq\langle\xi\rangle$ in $\operatorname{supp}\left(1-\varphi\left(t^{q}\langle\xi\rangle\right)\right)$. 
So we can write

$$
P=\left(\partial_{t}-i \lambda\right)\left(\partial_{t}+i \lambda\right)+r, \quad t^{1-\delta} r \in C\left([0, T] ; \Gamma_{s, A}^{1+1 / s}\right)
$$

and define

$$
U={ }^{t}\left(u_{1}, u_{2}\right), \quad u_{1}=\left(\partial_{t}+i \lambda\right) u, \quad u_{2}=\left\langle D_{x}\right\rangle u-m u_{1},
$$

$m(t, x, \xi)=\langle\xi\rangle / 2 i \lambda(t, x, \xi)$ to have that the equation $P u=0$ is equivalent to a first order $2 \times 2$ system $L U=0$,

$$
\begin{aligned}
& L=\partial_{t}+K\left(t, x, D_{x}\right), \quad K=D+R \\
& D=\left(\begin{array}{cc}
-i \lambda & 0 \\
0 & i \lambda
\end{array}\right), \quad t^{1-\delta} R \in C\left([0, T] ; \Gamma_{s, A}^{1 / s}\right) .
\end{aligned}
$$

Denoting by $\|u\|_{k, t, \mu}$ the norm of $u$ in $H^{k, t, \mu}\left(\boldsymbol{R}^{n}\right)$, it is

$$
C^{-1}\left(\|u(t)\|_{k, t, \mu+1}+\left\|\partial_{t} u(t)\right\|_{k, t, \mu}\right) \leq\|U(t)\|_{k, t, \mu} \leq C\left(\|u(t)\|_{k, t, \mu+1}+\left\|\partial_{t} u(t)\right\|_{k, t, \mu}\right),
$$

$0 \leq t \leq T, 0<k T^{\delta} / \delta \leq T_{0}, T_{0}$ the constant in Proposition 3.2, thus we prove Theorem 4 by the following result:

THEOREM 3.3. There are positive constants $k_{0}, C$ such that for every $U \in$ $C_{T}^{1}\left(H^{k, t, \mu+1}\right), k T^{\delta} / \delta \leq T_{0}, k \geq k_{0}$, which satisfies $L U=0$ we have

$$
\|U(t)\|_{k, t, \mu} \leq C\|U(0)\|_{k, 0, \mu}, \quad 0 \leq t \leq T .
$$

Proof. It is sufficient to prove (3.12) for $\mu=0$ since $\left\langle D_{x}\right\rangle^{\mu} L\left\langle D_{x}\right\rangle^{-\mu}$ satisfies the same hypotheses as $L$ and this is equivalent to prove

$$
\|V(t)\|_{0} \leq C\|V(0)\|_{0}, \quad 0 \leq t \leq T
$$

for every $V \in C\left([0, T] ; H^{1}\left(\boldsymbol{R}^{n}\right)\right) \cap C^{1}\left([0, T] ; H^{0}\left(\boldsymbol{R}^{n}\right)\right)$ such that $L_{\Lambda} V=0, L_{\Lambda}=$ $e^{\Lambda} L e^{-\Lambda}, \Lambda=\frac{k}{\delta}\left(T^{\delta}-t^{\delta}\right)\left\langle D_{x}\right\rangle^{1 / s}$.

From Proposition 3.2 and (3.11) we have

$$
L_{\Lambda}=\partial_{t}+k t^{-1+\delta}\left\langle D_{x}\right\rangle^{1 / s}+D+R_{1}, \quad t^{1-\delta} R_{1} \in C\left([0, T] ; S_{1,0}^{1 / s}\right), \quad k T^{\delta} / \delta \leq T_{0},
$$

so we can take $k$ large enough, say $k \geq k_{0}$, to make $k t^{-1+\delta}\left\langle D_{x}\right\rangle^{1 / s}+R_{1}(t)$ a positive operator while $D(t)+D^{*}(t)$ is uniformly bounded in $L^{2}\left(\boldsymbol{R}^{n}\right)$ for $0 \leq t \leq T$. This gives

$$
\frac{d}{d t}\|V(t)\|_{0}^{2} \leq C\|V(t)\|_{0}^{2}, \quad 0 \leq t \leq T \leq\left(\delta T_{0} / k\right)^{1 / \delta}
$$


for every $V \in C\left([0, T] ; H^{1}\left(\boldsymbol{R}^{n}\right)\right) \cap C^{1}\left([0, T] ; H^{0}\left(\boldsymbol{R}^{n}\right)\right)$ such that $L_{\Lambda} V=0$ which proves (3.13).

REMARK. It is possible to prove Theorem 4 also for the critical index $s=$ $q /(q-1)$. This needs the use of the Sharp Garding inequality as in the proof of Theorem 2 after an ad hoc version of Proposition 3.2 for more general functions $\Lambda$.

\section{Bibliography}

[1] Alinhac, S.: Remarks on the blowup rate of classical solutions to quasilinear multidimensional hyperbolic systems. J. Math. Pures Appl. 79, 8 (2000), 839-854.

[2] Cicognani, M.: A remark about hyperbolic equations with Log-Lipschitz coefficients. J. Partial Differ. Equations 10, 3 (1997), 284-288.

[ 3 ] Colombini, F.; Del Santo, D.; Kinoshita, T.: Well-posedness of the Cauchy problem for a hyperbolic equation with non-Lipschitz coefficients. Ann. Scuola Norm. Sup. Pisa Cl. Sci. 1 (2002), 327-358.

[ 4 ] Kajitani, K.: Cauchy problem for nonstrictly hyperbolic systems in Gevrey classes. J. of Math. of Kyoto Univ. 23 (1983), 599-616.

Massimo Cicognani

Dipartimento di Matematica

Università di Bologna

Piazza di Porta S. Donato, 5

40127 Bologna, Italy

and

Facoltà di Ingegneria II

Via Genova 181

47023 Cesena, Italy

e-mail: cicognan@dm.unibo.it 